\title{
Differentially expressed circulating microRNAs in Brazilian patients with COVID-19: a preliminary study on potential biomarkers for diagnosis and severity
}

Aline de Souza Nicoletti

UNICAMP

Marília Berlofa Visacri

UNICAMP

Carla Regina da Silva Correa da Ronda

UNICAMP

Pedro Eduardo do Nascimento Silva Vasconcelos

UNICAMP

Julia Coelho França Quintanilha

UNC Eshelman School of Pharmacy

Rafael Nogueira de Souza

UNICAMP

Deise de Souza Ventura

Hospital Estadual de Sumare

Adriana Eguti

Hospital Estadual de Sumare

Lilian Ferreira de Souza Silva

Hospital Estadual de Sumare

Mauricio Wesley Perroud Junior

UNICAMP

Rodrigo Ramos Catharino

UNICAMP

Leonardo Oliveira Reis

UNICAMP

Luiz Augusto dos Santos

Hospital Estadual de Paulinia

Nelson Durán

UNICAMP

Wagner José Fávaro

UNICAMP 


\section{Marcelo Lancellotti \\ UNICAMP \\ José Luiz da Costa}

UNICAMP

Patricia Moriel ( $\square$ patricia.moriel@fcf.unicamp.br)

UNICAMP

Eder de Carvalho Pincinato

UNICAMP

\section{Research Article}

Keywords: COVID-19, biomarkers, epigenomics, microRNAs

Posted Date: June 24th, 2021

DOI: https://doi.org/10.21203/rs.3.rs-630726/v1

License: (c) (1) This work is licensed under a Creative Commons Attribution 4.0 International License.

Read Full License 


\section{Differentially expressed circulating microRNAs in Brazilian patients with COVID-19: a preliminary study on potential biomarkers for diagnosis and severity}

Aline de Souza Nicoletti $1^{*}$, Marília Berlofa Visacri ${ }^{{ }^{*}}$, Carla Regina da Silva Correa da Ronda $^{2}$, Pedro Eduardo do Nascimento Silva Vasconcelos ${ }^{1}$, Julia Coelho França Quintanilha $^{3}$, Rafael Nogueira de Souza ${ }^{1}$, Deise de Souza Ventura ${ }^{4}$, Adriana Eguti ${ }^{4}$, Lilian Ferreira de Souza Silva ${ }^{4}$, Mauricio Wesley Perroud Junior ${ }^{1,4}$, Rodrigo Ramos Catharino $^{2,5}$, Leonardo Oliveira Reis ${ }^{6}$, Luiz Augusto dos Santos ${ }^{7}$, Nelson Durán ${ }^{8}$, Wagner José Fávaro ${ }^{8}$, Marcelo Lancellotti ${ }^{2}$, José Luiz da Costa ${ }^{2}$, Patricia Moriel ${ }^{2 \dagger}$, Eder de Carvalho Pincinato ${ }^{1}$.

${ }^{1}$ School of Medical Sciences, University of Campinas, Campinas, SP, Brazil.

${ }^{2}$ Faculty of Pharmaceutical Sciences, University of Campinas, Campinas, SP, Brazil.

${ }^{3}$ UNC Eshelman School of Pharmacy, University of North Carolina at Chapel Hill, Chapel Hill, NC, USA.

${ }^{4}$ Hospital Estadual Sumaré Dr. Leandro Francheschini, Sumaré, SP, Brazil.

${ }^{5}$ Innovare Biomarkers Laboratory, University of Campinas, Campinas, SP, Brazil.

${ }^{6}$ UroScience Laboratory, University of Campinas, Campinas, SP, Brazil.

${ }^{7}$ Hospital Municipal de Paulínia, Paulínia, SP, Brazil.

${ }^{8}$ Laboratory of Urogenital Carcinogenesis and Immunotherapy, University of

Campinas, Campinas, SP, Brazil.

*These authors contributed equally to this study.

${ }^{\dagger}$ Corresponding author: Patricia Moriel, PhD, Faculty of Pharmaceutical Sciences, University of Campinas, Cândido Portinari Street, 200, Cidade Universitária Zeferino Vaz - Barão Geraldo, Zip Code: 13083-871, Campinas, São Paulo, Brazil Tel: +551935218153; e-mail: patricia.moriel@ fcf.unicamp.br 


\begin{abstract}
Background: Coronavirus disease 2019 (COVID-19) is caused by a novel coronavirus, severe acute respiratory syndrome coronavirus 2 (SARS-CoV-2). It is known that host microRNAs (miRNAs) can be modulated to favor viral infection or to protect the host. Objective: The aim of this study was to identify differentially expressed circulating miRNAs in Brazilian patients with COVID-19 as potential biomarkers for diagnosis and severity. Methods: miRNAs were extracted from the blood plasma of eight patients with COVID-19 (four patients with mild/moderate COVID-19 and four patients with severe/critical COVID-19) and four healthy controls. The patients and controls were matched for sex and age. miRNA expression levels were detected using highthroughput sequencing. Differential miRNA expression and enrichment analyses were further evaluated. Results: A total of 18 human miRNAs were differentially expressed between patients with COVID-19 $(n=8)$ and controls $(n=4)$, with 13 significantly upregulated and five significantly downregulated miRNAs. miR-4433b-5p, miR-6780b3p, miR-6883-3p, miR-320b, miR-7111-3p, miR-4755-3p, miR-320c, and miR-6511a$3 \mathrm{p}$ were the most important miRNAs found significantly involved in the PI3K/AKT, Wnt/ $\beta$-catenin, and STAT3 signaling pathways, which have a crucial role in viral infections. Moreover, 42 miRNAs were differentially expressed between severe/critical patients with COVID-19 $(\mathrm{n}=4)$ and mild/moderate patients with COVID-19 $(\mathrm{n}=4)$. miR-451a, miR-101-3p, miR-185-5p, miR-30d-5p, miR-25-3p, miR-342-3p, miR-30e$5 p$, miR-150-5p, miR-15b-5p, and miR-29c-3p were the most important miRNAs found to be significantly involved in the $\mathrm{Wnt} / \beta$-catenin, NF- $\kappa \beta$, and STAT3 signaling pathways, which play crucial roles in immune response and inflammation.

Conclusions: Differentially expressed miRNAs found in this study may be used as potential biomarkers for the diagnosis and severity of COVID-19. Larger studies are needed to validate these miRNAs as biomarkers of COVID-19.
\end{abstract}

Keywords: COVID-19; biomarkers; epigenomics; microRNAs. 


\section{Introduction}

Coronavirus disease 2019 (COVID-19) is the name of the disease caused by the severe acute respiratory syndrome coronavirus 2 (SARS-CoV-2), whose initial outbreak occurred in Wuhan, China. Unfortunately, this virus has spread rapidly worldwide, culminating in the pandemic situation we have been experiencing since mid-March 2020 (1). SARS-CoV-2 is an RNA virus, which belongs to the genus Betacoronavirus, and is transmitted by inhalation of droplets containing the virus or by hand contact on surfaces containing the virus and subsequent contamination through the hands eyes, mouth, and nose. Symptoms include fever and dry cough in most patients, which may be accompanied by breathing difficulties, loss of taste, nausea, and diarrhea (2). Severe cases of COVID-19 can progress to severe acute respiratory syndrome (SARS) and the virus has also been reported to induce an intense inflammatory cascade, known as a cytokine storm (3). It is important to note that there is no specific treatment for the management of COVID-19 (4). Although some vaccines have been approved for emergency use by the Food and Drug Administration (FDA), such as the PfizerBioNTech, Moderna, and Janssen (Johnson \& Johnson) COVID-19 vaccines (5), and vaccination campaigns are beginning, some problems remain concerning: the slow availability of doses of these vaccines and the growing mutations undergone by the virus (6).

Real-time reverse transcriptase-polymerase chain reaction (RT-PCR) performed on nasopharyngeal or oropharyngeal swabs is the gold standard for the diagnosis of SARS-CoV-2. Although it is a specific and sensitive quantitative assay, there are some limitations, such as being relatively invasive and associated with an increased risk of cross-infection $(7,8)$. Other methods, such as virus antigen or serological antibody testing, may also be used to diagnose COVID-19. While rapid antigen tests detect SARS-CoV-2 in samples from the respiratory tract, rapid serological tests detect antibodies (IgM and/or IgG) produced over days to weeks after infection by the virus. Both tests are easy to handle and require minimal training. Rapid antibody tests, while less specific, can be useful for reducing exposure to risk factors during repeated sampling and are more cost effective compared to RT-PCR tests $(7,9)$. Computed tomography of the chest consists of a complementary examination for the diagnosis of COVID-19, which allows the monitoring of disease evolution $(7,10)$. In addition, the neutrophil-lymphocyte ratio (NLR) has been suggested as a prognostic marker for screening patients with COVID-19, with a higher NLR being associated with a poor prognosis (11). Thus, due to the several limitations of the techniques mentioned above, it is necessary to identify effective biomarkers for the diagnosis and severity of COVID19 , and the detection of microRNAs (miRNAs) in human samples may be an alternative.

miRNAs are defined as small, single-stranded, non-coding RNA molecules 2123 nucleotides in length that bind to the target transcript in the 3' untranslated region (UTR), inhibiting protein translation and destabilizing their target messenger RNAs (mRNAs). miRNAs can regulate almost a third of the human genome and are widely involved in multiple pathways, such as cell proliferation, cell death, stress resistance, and fat metabolism. In addition, evidence suggests that a gain or loss of function of one or more miRNAs is associated with the diagnosis, progression, and prognosis of several cancers and infectious diseases $(12,13)$.

In a recent systematic review focused on the description of miRNAs associated with SARS-CoV-2 infection in human cells, of the 29 studies included, only two of them reported the analysis of miRNA expression in patients with COVID-19 compared to non-COVID-19 samples (14). Most of the studies reported miRNA data based on 
genome sequencing of SARS-CoV-2 isolates and computational prediction analysis (14). In addition, our research group conducted a scoping review on miRNAs differentially expressed in SARS-CoV-2 infected animals and patients with COVID-19, excluding studies based only on in silico prediction analysis (15). Seven studies were included, six of which were conducted in patients, and we verified that miR-146a-5p, miR-21-5p, miR-15b-5p, and members of the let-7 family may serve as important biomarkers of COVID-19 (15). However, there have been few studies including populations from North and South America, where COVID-19 has a high incidence. Thus, the present study aimed to identify differentially expressed circulating miRNAs in Brazilian patients with COVID-19 as potential biomarkers for diagnosis and severity.

\section{Methods}

\subsection{Study approval}

The study was approved by the Research Ethics Committee of the School of Medical Sciences of the University of Campinas (UNICAMP) (numbers 36041420.0.000.5404 and 31049320.7.1001.5404). All participants or their guardians signed a consent form authorizing the use of their samples and data.

\subsection{Participants and eligibility criteria}

From May to September 2020, 12 participants were included in this study: four patients with severe/critical COVID-19 had been admitted to the Hospital Estadual Sumaré Dr. Leandro Francheschini (HES) in Sumaré city (SP, Brazil), affiliated with UNICAMP; four patients with mild/moderate COVID-19 had been admitted to the Hospital Municipal de Paulínia, in Paulínia city (SP, Brazil), and four healthy volunteers (controls).

The eligibility criteria were age $\geq 18$ years and admission to HES or Hospital Municipal de Paulínia with a positive result in SARS-CoV-2 nasopharyngeal swab RTPCR for patients with COVID-19, and age $\geq 18$ years and negative result in SARSCoV-2 nasopharyngeal swab RT-PCR for controls.

\subsection{Demographic and clinical data}

Data regarding the characteristics of patients with COVID-19 were obtained from medical records, including information concerning gender, age, race, and comorbidities. Additionally, they were classified by COVID-19 severity based on Falavigna et al., 2020 (16): mild, presence of any signs and symptoms of COVID-19, but without dyspnea or abnormal chest imaging; moderate, evidence of disease in the lower respiratory tract and $\mathrm{SpO}_{2}>93 \%$ on room air; severe, presence of one of the following factors: respiratory rate $>30 \mathrm{bpm}, \mathrm{SpO}_{2} \leq 93 \%$ on room air, $\mathrm{PaO}_{2} / \mathrm{FiO}_{2}<300 \mathrm{mmHg}$, pulmonary infiltrate $>50 \%$; and critical, respiratory failure, septic shock, and/or multiple organ dysfunction. Patients with severe/critical COVID-19 were also characterized by the length of hospital stay, drugs used for COVID-19 during hospitalization, the time they remained mechanically ventilated, and death. Data regarding control characteristics were obtained from interviews with volunteers, including information concerning age, gender, race, and comorbidities. Patients with severe/critical COVID-19, patients with mild/moderate COVID-19, and controls were matched for gender and age.

\subsection{Sample collection and miRNA extraction}


Whole blood was obtained from all participants. All patient samples were collected within ten days of diagnosis. Plasma was separated from whole blood by centrifugation at $2,500 \mathrm{rpm}, 4^{\circ} \mathrm{C}$ for $10 \mathrm{~min}$, and stored in a freezer at $-80^{\circ} \mathrm{C}$ until the experiments were carried out.

miRNA extraction was performed with $200 \mu \mathrm{L}$ of each plasma sample using the miRNeasy Serum/Plasma Kit (Qiagen, Cat No. 217184), following the manufacturer's instructions. At the end of the experiment, the samples were stored in a freezer at $-80^{\circ} \mathrm{C}$ until further use.

\subsection{Library construction and sequencing}

Library construction was performed with $5 \mu \mathrm{L}$ of each miRNA sample using the QIAseq ${ }^{\circledR}$ miRNA Library Kit (Qiagen, Cat No. 331502), following the manufacturer's instructions. For quality control of the samples, $1 \mu \mathrm{l}$ and $2 \mu \mathrm{l}$ of each miRNA sequencing library was analyzed using an Agilent Bioanalyzer and a Qubit fluorimeter, respectively, according to the manufacturer's instructions. A quality check of the raw data is provided in Appendix S1.

The samples were sent to the Life Sciences Core Facility (LaCTAD) from UNICAMP for sequencing. The library preparations were sequenced on an Illumina HiSeq 2500 platform, and 75 bp single-end reads were generated.

\subsection{Bioinformatics analysis}

Primary and secondary analyses were conducted in GeneGlobe. The primary analysis is based on counting the unique molecular identifiers (UMIs) and mapping the miRNA sequences, while the secondary analysis, using the UMI counts for each miRNA, performs the differential expression analysis. MiRWalk software was used to predict the miRNA target genes. MiRWalk provides predicted targets according to 12 different databases, including TargetScan (17). A matrix was constructed to identify the interaction between miRNAs and their predicted target genes, which were sorted according to the potential target genes of different miRNAs. For diagnostic analysis, genes related to five or more miRNAs were selected for enrichment analysis, using the Ingenuity Pathway Analysis (IPA ${ }^{\circledR}$, Qiagen) software to identify the main canonical signaling pathways involving differentially expressed miRNAs. For severity analysis, only target genes predicted by TargetScan and at least five different databases were selected for the following analyses. The genes targeted by at least two different miRNAs were selected for unsupervised enrichment analysis using the IPA software.

\subsection{Statistical analysis}

For the secondary analyses, the results were normalized using the DESeq2 method and the $p$-values listed were returned by the Bioconductor software packages, such that $p$-values less than 0.05 , were considered significant. The results were expressed as foldchange (FC) and fold-regulation (FR). FC is the normalized miRNA expression in each test sample divided by the normalized miRNA expression in the control sample. FR represents the FC results in a biologically meaningful way. FC values greater than one indicate up-regulation, and FR is equal to FC. FC values less than one indicate downregulation, and FR is the negative inverse of the FC. FR $\geq 1.6$, or FR $\leq-1.6$, were used as criteria to select differentially expressed miRNAs for the diagnosis of COVID-19. FR $\geq 2.0$, or FR $\leq-2.0$, were used as criteria to select miRNAs differentially expressed for COVID-19 severity. 
For enrichment analysis (the most important canonical signaling pathways) of the predicted target genes, $p$-values were calculated by Fisher's exact test, and $p$-values less than 0.05 , were considered significant.

\section{Results}

\subsection{Characteristics of participants}

Twelve participants were included in this study: patients with mild/moderate COVID-19 ( $\mathrm{n}=4$; sex: 2 male/2 female; mean age \pm standard deviation (SD): $61.8 \pm$ 11.7 years), patients with severe/critical COVID-19 $(\mathrm{n}=4$; sex: 2 male/ 2 female; mean age \pm SD: $64.0 \pm 8.6$ years $)$, and controls $(n=4$; sex: 2 male/ 2 female; mean age \pm SD: $62.8 \pm 14.9$ years). Sex and age were similar among the three groups. Table 1 shows the detailed characteristics of the participants.

Table 1. Characteristics of patients with COVID-19 and controls.

\begin{tabular}{|c|c|c|c|c|}
\hline ID & Gender & Age (y) & Race & Comorbidities \\
\hline \multicolumn{5}{|c|}{ Mild/moderate COVID-19 } \\
\hline MM1 & Female & 47 & NA & $\begin{array}{l}\text { Systemic arterial hypertension } \\
\text { Diabetes }\end{array}$ \\
\hline MM2 & Male & 58 & NA & None \\
\hline MM3 & Female & 69 & NA & None \\
\hline MM4 & Male & 73 & NA & Systemic arterial hypertension \\
\hline \multicolumn{5}{|c|}{ Severe/critical COVID-19 } \\
\hline $\mathrm{SC} 1$ & Female & 55 & Caucasian & $\begin{array}{l}\text { Systemic arterial hypertension } \\
\text { Diabetes } \\
\text { Congestive heart failure } \\
\text { Chronic kidney disease }\end{array}$ \\
\hline $\mathrm{SC} 2$ & Male & 59 & Non-caucasian & None \\
\hline SC3 & Female & 68 & Caucasian & $\begin{array}{l}\text { Systemic arterial hypertension } \\
\text { Diabetes } \\
\text { Hypothyroidism } \\
\text { Obesity }\end{array}$ \\
\hline $\mathrm{SC} 4$ & Male & 74 & Non-caucasian & $\begin{array}{l}\text { Systemic arterial hypertension } \\
\text { Diabetes } \\
\text { Chronic kidney disease }\end{array}$ \\
\hline \multicolumn{5}{|c|}{ Controls } \\
\hline HV1 & Female & 45 & Caucasian & None \\
\hline HV2 & Male & 56 & Caucasian & None \\
\hline HV3 & Female & 73 & Caucasian & Arrhythmia \\
\hline HV4 & Male & 77 & Caucasian & $\begin{array}{l}\text { Previous myocardial infarction and } \\
\text { stroke }\end{array}$ \\
\hline
\end{tabular}

Regarding patients with severe/critical COVID-19, the average length of stay was $36 \pm 27$ days; all patients received amoxicillin + clavulanate (1 g IV t.i.d.) and azithromycin (500 mg PO q.d.); two patients also received oseltamivir (75 mg PO b.i.d.) as antimicrobial treatment, two patients used dexamethasone (6 mg q.d.) to treat inflammation, and all patients were treated with anticoagulants (enoxaparin 40-60 mg/d 
SC or heparin 10,000-15,000 UI/d SC); they remained mechanically ventilated for 515 \pm 346 hours; and two patients died.

\subsection{MiRNAs as biomarkers of diagnosis for COVID-19}

\subsubsection{Differential miRNA expression profiling}

A total of 18 miRNAs were differentially expressed between patients with COVID19 and the controls, of which 13 were significantly upregulated and five were significantly downregulated (Figure 1 and Table 2).

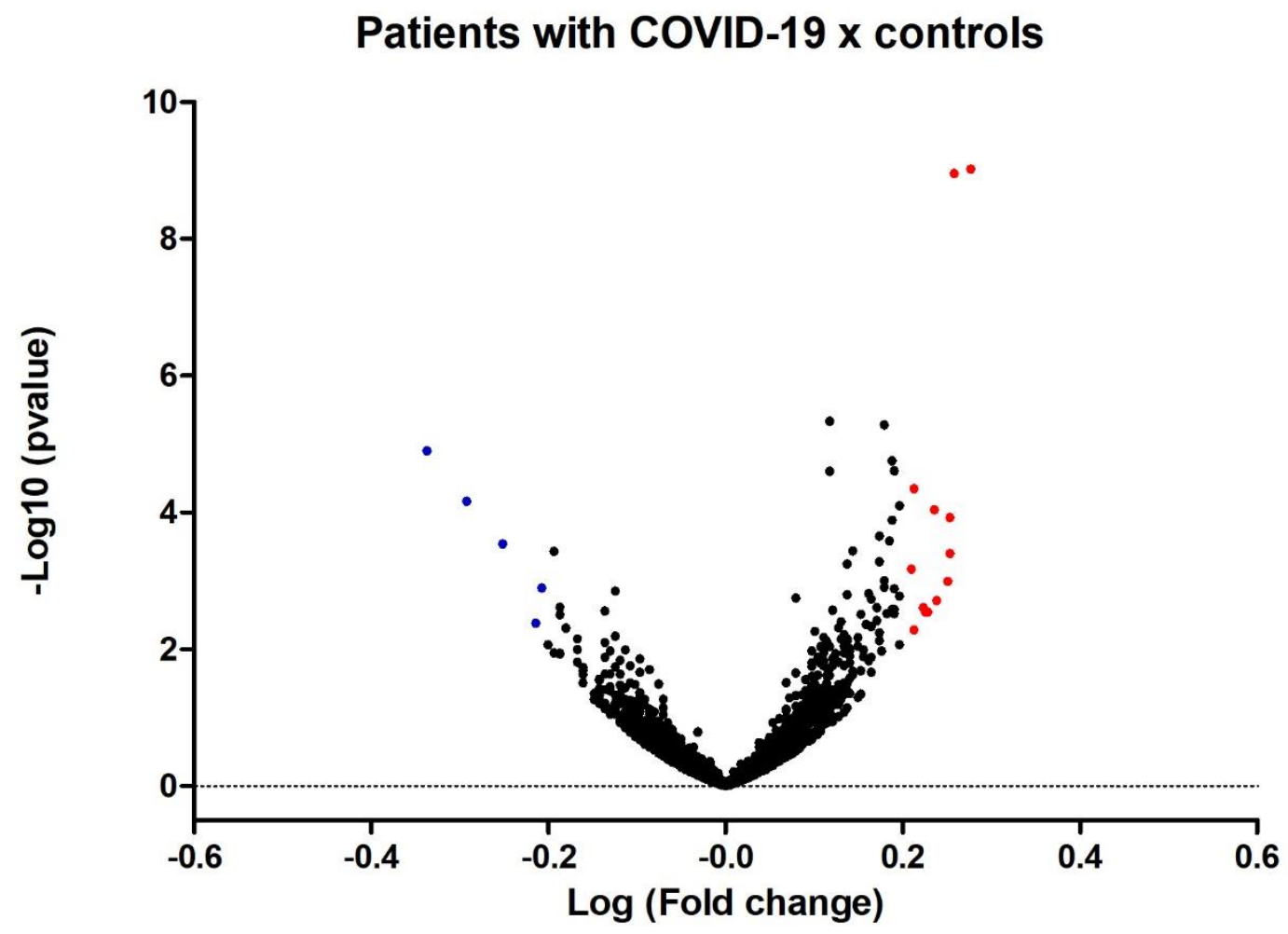

Figure 1. Differential miRNA expression between patients with COVID-19 and controls (healthy volunteers). The abscissa presents the logarithmic value, logFC, of multiple differences in gene expression between the two groups and the ordinate represents the negative pair value of the $p$-value of the change in gene expression. Each point in the figure represents an miRNA. miRNAs with significant differences are represented by blue (downregulated) and red (upregulated) dots. miRNAs without significant differences are represented by black dots.

The expression of miR-6780b-3p, miR-6883-3p, miR-4769-5p, miR-6873-3p, miR320b, miR-7111-3p, miR-4755-3p, miR-320c, miR-6511a-3p, miR-320d, miR-5187-3p, miR-4508, and miR-4659a-5p was at least 1.6-fold higher in patients with COVID-19 than in controls. In addition, the expression of miR-4433b-5p, miR-16-2-3p, miR126$3 p$, miR-150-5p, and miR-224-5p was reduced by more than 1.6 -fold in patients with COVID-19 compared with that in controls (Table 2). 
Table 2. Differentially expressed miRNAs between patients with COVID-19 and controls $(F R \geq 1.6$ or $F R \leq-1.6)$.

\begin{tabular}{|c|c|c|c|}
\hline miRNA & FR & FC & $P$ value \\
\hline \multicolumn{4}{|l|}{ Upregulated } \\
\hline miR-6780b-3p & 1.89 & 1.89 & $<0.001$ \\
\hline miR-6883-3p & 1.81 & 1.81 & $<0.001$ \\
\hline miR-4769-5p & 1.79 & 1.79 & $<0.001$ \\
\hline miR-6873-3p & 1.79 & 1.79 & $<0.001$ \\
\hline miR-320b & 1.78 & 1.78 & 0.001 \\
\hline miR-7111-3p & 1.73 & 1.73 & 0.002 \\
\hline miR-4755-3p & 1.72 & 1.72 & $<0.001$ \\
\hline miR-320c & 1.69 & 1.69 & 0.003 \\
\hline miR-6511a-3p & 1.68 & 1.68 & 0.003 \\
\hline miR-320d & 1.67 & 1.67 & 0.002 \\
\hline miR-5187-3p & 1.63 & 1.63 & $<0.001$ \\
\hline miR-4508 & 1.63 & 1.63 & 0.005 \\
\hline miR-4659a-5p & 1.62 & 1.62 & $<0.001$ \\
\hline \multicolumn{4}{|l|}{ Downregulated } \\
\hline miR-4433b-5p & -2.15 & 0.46 & $<0.001$ \\
\hline miR-16-2-3p & -1.97 & 0.51 & $<0.001$ \\
\hline miR-126-3p & -1.77 & 0.56 & $<0.001$ \\
\hline miR-150-5p & -1.65 & 0.61 & 0.004 \\
\hline miR-224-5p & -1.61 & 0.62 & 0.001 \\
\hline
\end{tabular}

Abbreviations: FC, fold change; FR, fold regulation 
Additional analysis of only patients with mild/moderate COVID-19 compared to controls and only patients with severe/critical COVID-19 compared to controls were performed (Appendix S2 to S4). Only eight miRNAs were differentially expressed in the three analyses (Table 2 and Appendix S2): miR-4433b-5p, miR-6780b-3p, miR6883-3p, miR-320b, miR-7111-3p, miR-4755-3p, miR-320c, and miR-6511a-3p. Figure 2 shows a comparison of the expression levels of these miRNAs between the groups.
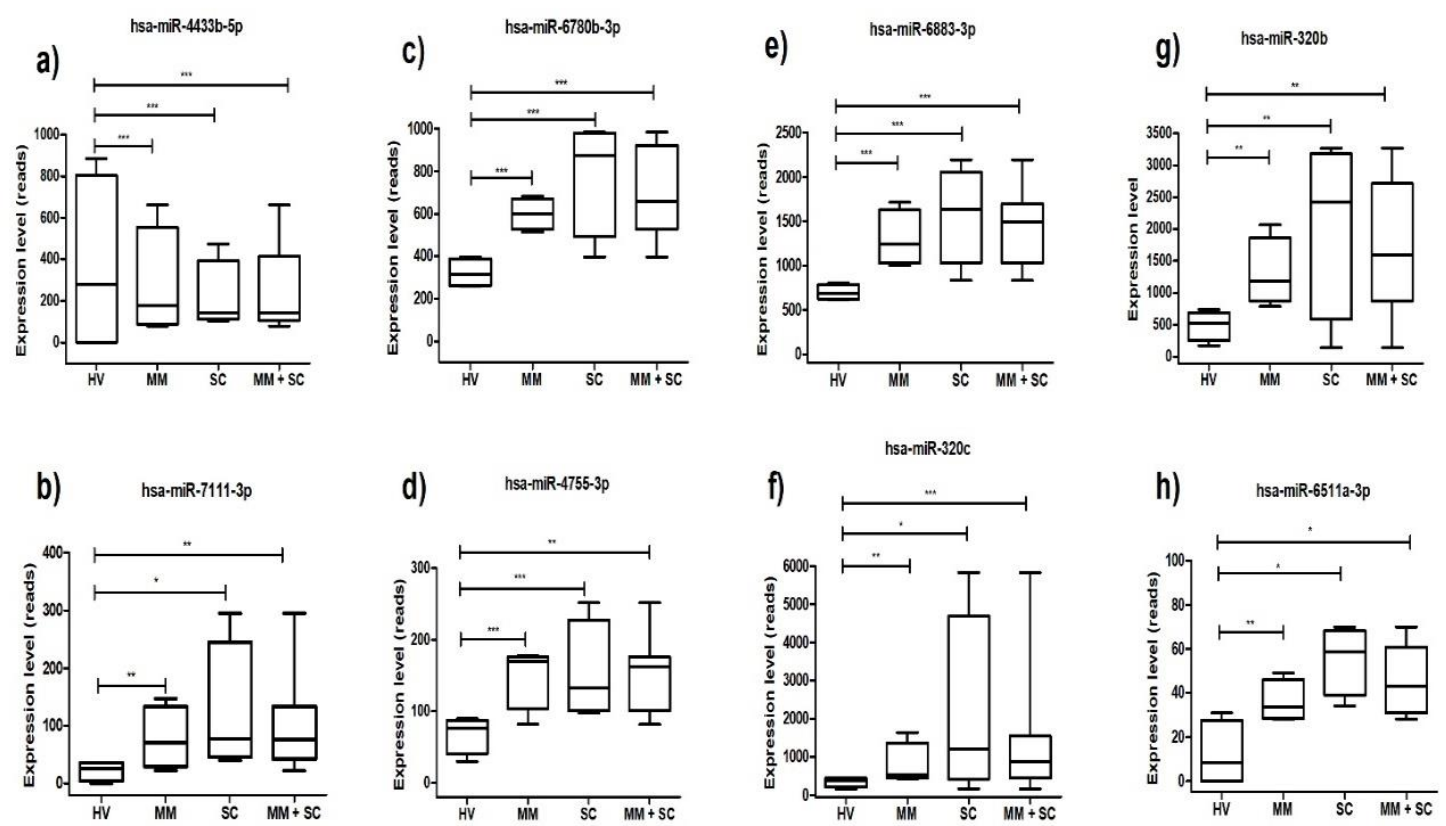

Figure 2. Expression of miRNAs in each sample. The abscissa presents the status of each participant: healthy volunteer $(\mathrm{HV})(\mathrm{n}=4)$, patient with mild/moderate COVID-19 $(\mathrm{MM})(\mathrm{n}=4)$, patient with severe/critical COVID-19 $(\mathrm{SC})(\mathrm{n}=4)$ and $\mathrm{MM}+\mathrm{SC}(\mathrm{n}=$ 8). The letters b, c, d, and h represent miRNAs consistently upregulated. The letters a and e represent miRNAs consistently downregulated and the letters $f$ and $g$ represent miRNAs only downregulated in severe patients. Boxplot features: minimum whisker, the smallest value within; minimum box, $25^{\text {th }}$ percentile; center, median; maximum box, $75^{\text {th }}$ percentile; maximum whisker, the largest value within. ${ }^{* * *} \mathrm{p} \leq 0.001 ; * * \mathrm{p} \leq 0.01$; $* \mathrm{p} \leq 0.05$.

\subsubsection{Enrichment analysis of differentially expressed miRNA target genes}

After the identification of the eight miRNAs cited above as potential diagnostic biomarkers, enrichment analysis was performed, and the top 50 canonical signaling pathways are shown in Figure 3. Of the 50 most enriched signaling pathways, three appear to have an important role in modulating viral infections by miRNAs: Wnt $/ \beta$ catenin signaling, phosphoinositide 3-kinase/protein kinase B (PI3K/AKT) signaling, and the STAT3 pathway. A total of 242 genes were related to these pathways, and 64 of them were common to at least two of the above-mentioned pathways (data not shown). The matrix constructed for the analysis of miRNA-gene interactions indicated that miR$320 \mathrm{~b}$ and miR-320c interacted the most with the 242 selected genes, and their interactions are described in more databases. 


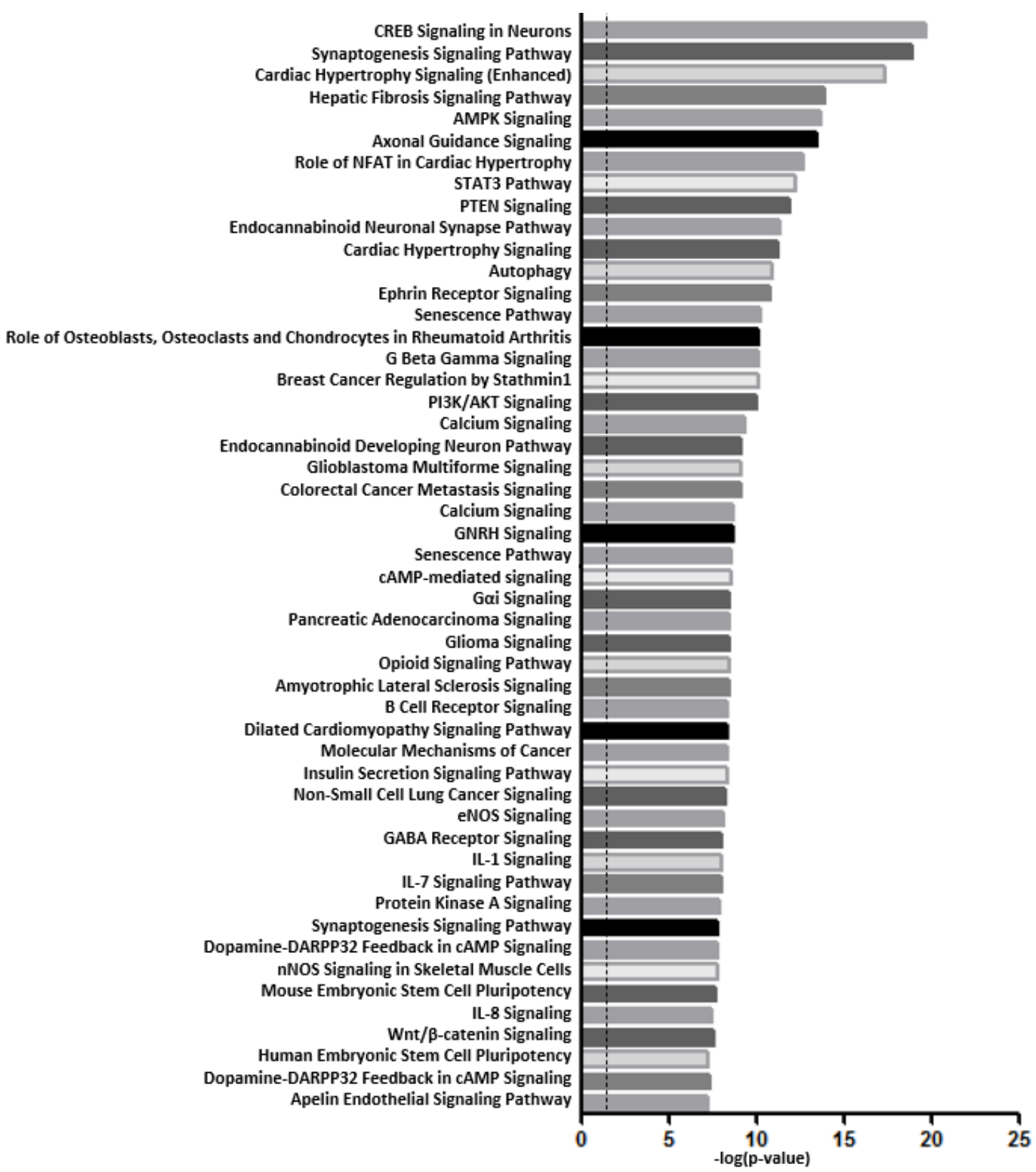

Figure 3. Enrichment analysis of the predicted target genes (top 50 canonical signaling pathways). Enrichment analysis performed by Ingenuity Pathway Analysis (IPA $^{\circledR}$, Qiagen bioinformatics). The dashed line represents $-\log (p$-value $)=1.3$ or $p$ value $=0.05$ (Fisher's exact test) .

\subsection{MiRNAs as biomarkers of severity of COVID-19}

\subsubsection{Differential miRNA expression profiling}

A total of 42 miRNAs were differentially expressed between severe/critical patients with COVID-19 and mild/moderate COVID-19 patients, all at least 2-fold lower in patients with severe or critical COVID-19 than in patients with mild/moderate COVID19 (Figure 4 and Table 3). miR-451a, miR-101-3p, miR-185-5p, miR-30d-5p, miR-25$3 p$, miR-342-3p, miR-30e-5p, miR-150-5p, miR-15b-5p, and miR-29c-3p were the 10 most downregulated miRNAs (Table 3 ). 
Patients with severe/critical COVID-19 x patients with mild/moderate COVID-19

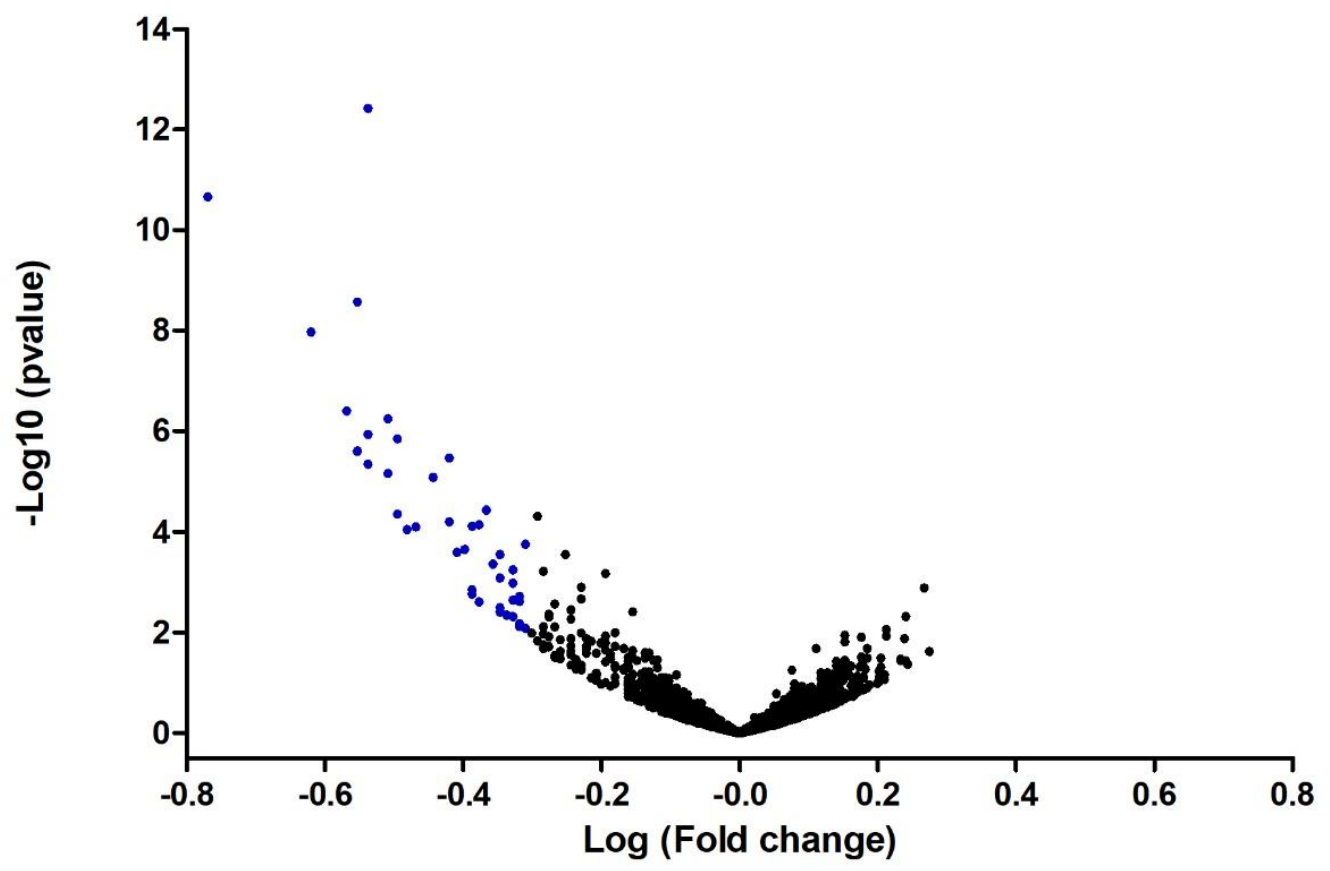

Figure 4. Differential miRNA expression between patients with severe/critical and those with mild/moderate COVID-19. The abscissa presents the logarithmic value, $\log \mathrm{FC}$, of the multiple differences in gene expression between the two groups, and the ordinate presents the negative pair value of the $p$-value of the change in gene expression. Each point in the figure represents an miRNA. miRNAs with significant differences are represented by blue (downregulated) and red (upregulated) dots. miRNAs without significant differences are represented by black dots. 
Table 3. Differentially expressed miRNAs between patients with severe/critical COVID-19 and in those with mild/moderate COVID-19 (FR $\geq 2.0$ or FR $\leq-\mathbf{2 . 0})$

\begin{tabular}{|c|c|c|c|}
\hline miRNA & FR & FC & $P$ value \\
\hline miR-451a & -5.79 & 0.17 & $<0.001$ \\
\hline miR-101-3p & -4.15 & 0.24 & $<0.001$ \\
\hline miR-185-5p & -3.74 & 0.27 & $<0.001$ \\
\hline miR-30d-5p & -3.58 & 0.28 & $<0.001$ \\
\hline miR-25-3p & -3.56 & 0.28 & $<0.001$ \\
\hline miR-342-3p & -3.55 & 0.28 & $<0.001$ \\
\hline miR-30e-5p & -3.49 & 0.29 & $<0.001$ \\
\hline miR-150-5p & -3.47 & 0.29 & $<0.001$ \\
\hline miR-15b-5p & -3.4 & 0.29 & $<0.001$ \\
\hline miR-29c-3p & -3.26 & 0.31 & $<0.001$ \\
\hline miR-10b-5p & -3.18 & 0.31 & $<0.001$ \\
\hline miR-16-2-3p & -3.17 & 0.32 & $<0.001$ \\
\hline miR-186-5p & -3.11 & 0.32 & $<0.001$ \\
\hline miR-16-5p & -3.05 & 0.33 & $<0.001$ \\
\hline miR-425-5p & -2.97 & 0.34 & $<0.001$ \\
\hline miR-187-3p & -2.76 & 0.36 & $<0.001$ \\
\hline miR-125a-5p & -2.66 & 0.38 & $<0.001$ \\
\hline miR-106b-3p & -2.62 & 0.38 & $<0.001$ \\
\hline miR-22-3p & -2.55 & 0.39 & $<0.001$ \\
\hline miR-144-5p & -2.48 & 0.4 & $<0.001$ \\
\hline miR-151a-3p & -2.44 & 0.41 & 0.001 \\
\hline miR-30a-5p & -2.43 & 0.41 & $<0.001$ \\
\hline miR-92a-3p & -2.43 & 0.41 & 0.002 \\
\hline miR-15a-5p & -2.38 & 0.42 & $<0.001$ \\
\hline miR-195-5p & -2.36 & 0.42 & 0.002 \\
\hline $\mathrm{miR}-4508$ & -2.35 & 0.43 & $<0.001$ \\
\hline miR-194-5p & -2.26 & 0.44 & $<0.001$ \\
\hline miR-140-3p & -2.25 & 0.45 & 0.003 \\
\hline miR-142-5p & -2.24 & 0.45 & 0.004 \\
\hline miR-99a-5p & -2.24 & 0.45 & $<0.001$ \\
\hline miR-363-3p & -2.23 & 0.45 & $<0.001$ \\
\hline let- $7 g-5 p$ & -2.18 & 0.46 & 0.005 \\
\hline miR-20a-5p & -2.14 & 0.47 & 0.002 \\
\hline miR-144-3p & -2.13 & 0.47 & $<0.001$ \\
\hline miR-10a-5p & -2.12 & 0.47 & 0.001 \\
\hline miR-378a-3p & -2.12 & 0.47 & 0.005 \\
\hline let-7f-5p & -2.1 & 0.48 & 0.008 \\
\hline miR-660-5p & -2.08 & 0.48 & 0.007 \\
\hline miR-3135b & -2.07 & 0.48 & 0.002 \\
\hline miR-96-5p & -2.07 & 0.48 & 0.002 \\
\hline miR-125b-5p & -2.05 & 0.49 & $<0.001$ \\
\hline let-7i-5p & -2.04 & 0.49 & 0.008 \\
\hline
\end{tabular}

As cited above, an analysis of patients with severe/critical COVID-19 compared to controls was also performed (Appendix S2 and S4).

\subsubsection{Enrichment analysis of differentially expressed miRNA target genes}

After the identification of 10 miRNAs cited as potential biomarkers of severity of COVID-19, enrichment analysis was performed, and the most important canonical signaling pathways are shown in Figure 5. Three pathways, Wnt/ $\beta$-catenin, NF- $\kappa \beta$, and STAT3 signaling, play an important role in viral infection and inflammatory response. A total of 159 genes were related to these pathways, and 34 of them were common to at least two of the above-mentioned pathways (data not shown). From the analysis of miRNA-gene interactions, miR-185-5p and miR-15b-5p are the ones that most interact with the 159 selected genes, and their interactions are described in more databases. 


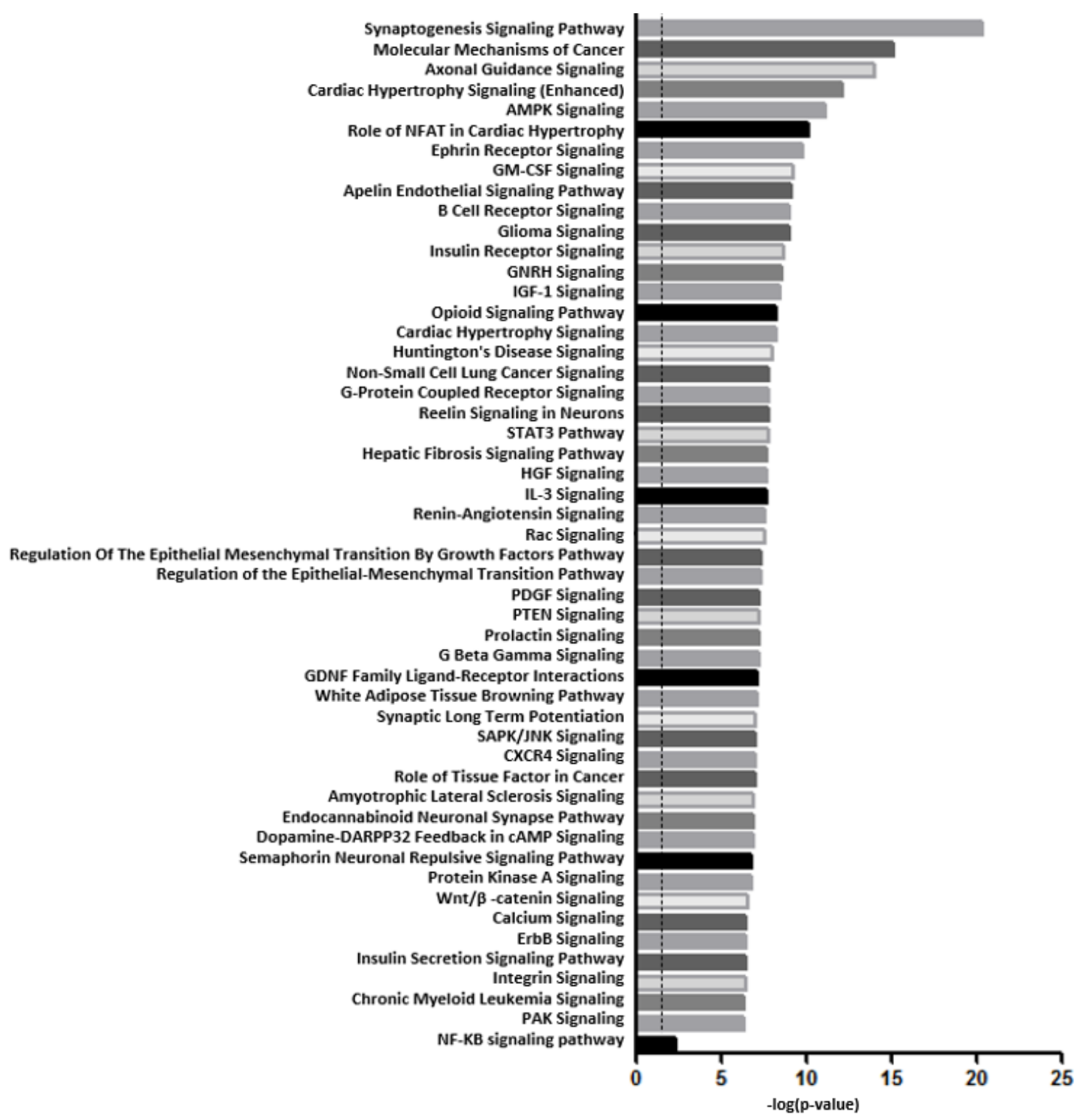

Figure 5. Enrichment analysis of the predicted target genes (most important canonical signaling pathways). Enrichment analysis performed by Ingenuity Pathway Analysis (IPA ${ }^{\circledR}$, Qiagen bioinformatics). The dashed line represents $-\log (p$-value $)=1.3$ or $p$-value $=0.05$ (Fisher's exact test).

\section{Discussion}

miRNAs that enable the identification of SARS-CoV-2 infection may directly target the viral genome and proteins associated with the entry of the virus into the cell (e.g., ACE2, ADAM17, TMPRSS2), as well as regulate the immune system (18). Based on this, a study suggested some miRNAs that could be important for COVID-19 diagnosis, such as miR-15b-5p, miR-195-5p, miR-221-3p, and miR-140-3p (18). In our study, we identified eight important miRNAs as potential diagnostic biomarkers (miR-4433b-5p, miR-6780b-3p, miR-6883-3p, miR-320b, miR-7111-3p, miR-4755-3p, miR-320c, and miR-6511a-3p) that were not described in previously published articles on SARS-CoV2 infected animals and patients with COVID-19 (15). However, in a recent study, the most downregulated miRNA in our study, miR-4433b-5p, was differentially expressed between moderate and severe COVID-19 cases, suggesting that this miRNA might be a 
potential candidate for stratifying patients based on severity (19). In our study, miR$4433 b-5 p$ was downregulated in both mild/moderate and severe/critical cases compared to controls; however, miR-4433b-5p was not differentially expressed between severe/critical cases and mild/moderate cases.

In addition, the expression of miR-16-2-3p was downregulated in patients with COVID-19 compared to controls, contrary to the study by Li et al., in which the expression was upregulated (20). Moreover, miR-16-2-3p was found to be positively regulated in cells infected with SARS-CoV-2 compared to samples of normal human lung tissue (21). High levels of this miRNA may play a potential role in mediating SARS-COV-2 infection (14). However, the differential expression of miR-16-2-3p in patients with COVID-19 does not appear to be specific, since it was found in the comparison between patients with recent or complicated type 2 diabetes (22). In addition, miR-16-2-3p expression was found to be upregulated in the plasma of patients with non-syndromic cleft lip (23) and downregulated in resistant glucocorticoid patients with ulcerative colitis (24).

We also found that miR-126-3p was differentially expressed and downregulated in patients with COVID-19 compared with that in controls, corroborating the findings of Sabbatinelli et al. (25) and Garg et al. (26); however, these studies compared only patients with severe COVID-19 with healthy volunteers. Although we found miR-126$3 p$ as a potential diagnostic biomarker in our study, miR-126-3p downregulation was

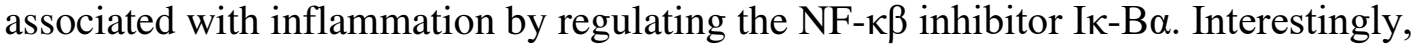
miR-126-3p has also been associated with endothelial dysfunction, which can partially explain the vascular damage observed in the lungs of patients with COVID-19, associated with the presence of intracellular viruses and perivascular T-cell infiltrates (27), contributing to disease severity.

In the comparison between patients with mild/moderate COVID-19 and controls, we found that miR-99a-5p and miR-378a-3p were upregulated. In a study by Tang et al., miR-99a-5p was found to be downregulated in severe cases compared to healthy volunteers and in severe cases compared to moderate cases, and was associated with the expression of the proinflammatory genes IGF1R and MTMR3, which induce weaker antiviral immunity (28). In addition, the increase in miR-378a-3p expression attenuated hypoxia-induced lesions in cardiomyocytes by suppressing the NEAT1 lncRNA (29), although in a study by Chen et al., elevated levels of miR-378a-3p were associated with a poor prognosis for COVID-19 (30).

In our study, from the eight most important miRNAs for the diagnosis of COVID-19, a total of 242 target genes were found to be related to the Wnt/ $\beta$-catenin signaling, PI3K/AKT signaling, and STAT3 pathways, which according to Barbu et al. are well known to be involved in viral infection. Wnt represents a group of highly preserved pathways in vertebrates through which extracellular signals are transported into the cell. In addition, there appears to be a relationship between the Wnt pathway and innate immune response in the host. However, there are theories that type I interferon (IFN) signaling can be increased while inhibiting the Wnt pathway (31). STAT3 is a member of the STAT protein family that controls the expression of genes regulating different biological processes, such as immune responses, inflammation, and apoptosis. STAT3 protein is present in the cytoplasm in an inactive form and is activated by numerous types of cytokines, especially interleukin 6 (IL-6), which is a predictive marker of severity in COVID-19. Furthermore, STAT3 may have multiple roles during SARS-CoV-2 infection, including induction of pro-inflammatory responses, promotion of a cytokine storm, impairment of antiviral immune responses, alteration of virus replication, and exacerbation of lymphopenia (32). Finally, with 
regard to the PI3K/AKT signaling pathway, it has an important role in viral replication, and its activation has been reported in many viruses, such as hepatitis B and C and human immunodeficiency. In addition, Shwetha et al. demonstrated that the upregulation of miR-320c during hepatitis $\mathrm{C}$ virus infection targets P13K/AKT signaling, which increases cell survival and corroborates the results found in our study $(31,33)$. Therefore, these signaling pathways may be dysregulated in patients with COVID-19, thus contributing to its pathogenesis.

miRNAs as biomarkers of COVID-19 severity may be associated with the host immune response and inflammation, and may serve as potential therapeutic targets (28). Our study showed that miR-451a, miR-101-3p, miR-185-5p, miR-30d-5p, miR-25-3p, miR-342-3p, miR-30e-5p, miR-150-5p, miR-15b-5p, and miR-29c-3p were the ten most important downregulated miRNAs as potential biomarkers of disease severity. Among these miRNAs, miR-15b-5p was found to be differentially expressed in two previous studies $(18,28)$. According to Kim et al., miR-15b-5p directly binds to the viral genome, and in their study, it was also found to be downregulated during SARS-CoV-2 infection, which may allow the virus to escape the host immune defense by inhibiting apoptosis and promoting the proliferation of infected cells (18). However, according to Tang et al., its upregulation can accelerate viral replication, mediate virus-induced changes in the cell transcriptome, and intensify the severity of COVID-19 (28). miR$15 \mathrm{~b}-5 \mathrm{p}$ was also predicted by three independent studies included in the systematic review published by Marchi et al., indicating that this miRNA can act by regulating host and SARS-CoV-2 genes (14).

The type I IFN signaling pathway is involved in viral infections (31). Recent studies have shown that IFN is crucial for the severity of COVID-19, and its deficiency can lead to severe disease $(34,35)$. However, the type I IFN pathway was not significantly enriched in the ten downregulated miRNAs in severe/critical COVID-19 patients. On the other hand, our study showed that these downregulated miRNAs were involved in the $\mathrm{Wnt} / \beta$-catenin signaling pathway, an immune regulation pathway that may be hyperactivated. Once type I IFN signaling can be increased while inhibiting the Wnt pathway (29), it is possible that the hyperactivated Wnt signaling pathway is related to decreased type I IFN signaling pathway. Moreover, NF- $\kappa \beta$ and STAT3 signaling pathways are significantly enriched, and they are also known to be involved in viral infection (31). It is known that the severity of COVID-19 is dependent on a cytokine storm, most likely induced by the IL- 6 amplifier, which is a hyperactivation machinery that regulates the NF- $\kappa B$ pathway and is stimulated by the simultaneous activation of STAT3 and NF- $\kappa$ B signaling in non-immune cells, including alveolar epithelial cells and endothelial cells (36). Therefore, the hyperactivation of these two signaling pathways plays a key role in the inflammation of non-immune cells and may cause fatal symptoms such as acute respiratory distress syndrome (ARDS), severe pneumonia, multiorgan failure, and coagulation (36). The signaling pathways found may be potential therapeutic targets for the treatment of patients with severe or critical COVID-19.

In addition to the ten most downregulated miRNAs found in this study, miR195-5p, miR-140-5p, miR-144-3p, miR-125a-5p, miR-30a-5p, let-7f-5p, let-7g-5p, and let-7i-5p were also downregulated when comparing patients with severe/critical to mild/moderate COVID-19, and miR-195-5p, miR-144-3p, miR-125a-5p, miR-30a-5p, miR-21-5p, let-7a-5p, let-7d-5p, and let-7f-5p when comparing patients with severe/critical COVID-19 to controls. Thus, they appear to be related to the disease severity. According to Kim et al., miR-195-5p directly binds to the viral genome, but unlike our results, this miRNA was upregulated in SARS-CoV-2 infection and appears 
to be related to the promotion of apoptosis by inducing cell cycle arrest and the prevention of excessive proliferation of infected cells (18). Moreover, miR-140-3p and miR-144-3p were found to be downregulated in the studies by Kim et al. and Li et al., respectively, as well as in our work $(18,20)$. It is known that miR-140-3p targets TMPRSS2, and its downregulation can contribute to viral infection by inhibiting apoptosis and promoting cell proliferation (18). The pathophysiological implication of miR-144-3p dysregulation has not been reported by Li et al. (20). In contrast, Guo et al. showed that this miRNA is upregulated in the lung tissues of mice infected with the H7N9/AH1-PB2-627E/701N strain of the influenza virus (37). Furthermore, downregulation of miR-125a-5p and miR-30 may favor entry of the virus into the cell. miR-125a-5p was predicted to directly target ACE2 mRNA or associated ACE2 pathways, while miR-30a was found to be negatively correlated with the TMPRSS2 protein (14). According to Tang et al. and corroborating our results and those of Sabatinelli et al., the downregulation of miR-21-5p is associated with inflammation (25, 28), while its upregulation appears to be associated with cardiac fibrosis (26). Finally, the altered expression of members of the let-7 family found in our work supports the findings of Chen et al. and Zheng et al., revealing the importance of this family in the activation of $T$ cells and during the inflammatory response $(30,38)$. Indeed, let-7 miRNAs play a key role in the activation of the immune system and the inflammatory response by targeting the IL-6 gene and reducing its expression $(39,40)$.

This study has some limitations: (a) the blood samples were collected at different times after diagnosis of COVID-19, i.e., different periods of infection between patients; (b) we did not include non-COVID-19 patients with respiratory viral infection as positive controls (e.g., patients infected with influenza virus) to validate the specificity of these miRNAs as biomarkers of COVID-19; (c) we also did not include asymptomatic COVID-19 patients, and thus, it is not known if the differential expression of these miRNAs would be useful for diagnosing these patients; (d) we do not know which strains of SARS-CoV-2 infected the patients studied; (e) although we matched age and gender between healthy volunteers and patients with COVID-19, other comorbidities such as type 2 diabetes, obesity and coronary artery diseases that are known to alter the expression of circulating miRNAs could not be precisely combined in our study; and (f) a small number of patients were used in the present study; however, the next step of our research will be the validation of the main miRNAs found by RTPCR (preferred method for the identification of miRNAs) with a large cohort.

In this study, we aimed to identify differentially expressed circulating miRNAs in patients with COVID-19 as potential biomarkers for diagnosis and severity of the disease. To the best of our knowledge, this is the first Brazilian study to address this objective. The main findings were as follows: (a) miR-4433b-5p, miR-6780b-3p, miR6883-3p, miR-320b, miR-7111-3p, miR-4755-3p, miR-320c, and miR-6511a-3p were the most important miRNAs as diagnostic biomarkers. These miRNAs are involved in the PI3K/AKT, Wnt/ $\beta$-catenin, and STAT3 signaling pathways, which play a crucial role in viral infections; and (b) miR-451a, miR-101-3p, miR-185-5p, miR-30d-5p, miR25-3p, miR-342-3p, miR-30e-5p, miR-150-5p, miR-15b-5p, and miR-29c-3p were the most important miRNAs identified as biomarkers of disease severity. These miRNAs are involved in the $\mathrm{Wnt} / \beta$-catenin, NF- $\kappa \beta$, and STAT3 signaling pathways, which play a crucial role in the immune response and inflammation.

\section{Conclusion}


In conclusion, the differentially expressed miRNAs identified in this study may be used as potential biomarkers for the diagnosis and severity of COVID-19. Moreover, these miRNAs and signaling pathways may be potential targets for developing therapeutics for treating COVID-19. Larger studies are needed to validate these miRNAs as biomarkers of COVID-19.

\section{Funding Statement}

This study was financed in part by the Coordenação de Aperfeiçoamento de Pessoal de Nível Superior - Brasil (CAPES) [Finance Code 001 and 88881.504454/2020-01]. ASN is a recipient of a doctoral scholarship from the Coordenação de Aperfeiçoamento de Pessoal de Nível Superior - Brasil (CAPES) [number 88887.511334/2020-00]. MBV is a recipient of a postdoctoral scholarship from the Coordenação de Aperfeiçoamento de Pessoal de Nível Superior - Brasil (CAPES) [number 88887.504453/2020-00].

\section{Acknowledgments}

We thank the staff of the Life Sciences Core Facility (LaCTAD) from University of Campinas (UNICAMP), for the quality control of the samples and sequencing.

\section{Abbreviations}

COVID-19: Coronavirus disease 2019

SARS-CoV-2: Severe acute respiratory syndrome coronavirus 2

SARS: Severe acute respiratory syndrome

FDA: Food and Drug Administration

RT-PCR: Real-time reverse transcriptase-polymerase chain reaction

NLR: Neutrophil-lymphocyte ratio

miR: microRNA

miRNAs: microRNAs

UTR: Untranslated region

mRNAs: messenger RNAs

$\mathrm{SpO}_{2:}$ Peripheral $\mathrm{O}_{2}$ saturation

Bpm: Beats per minute

$\mathrm{PaO}_{2} / \mathrm{FiO}_{2}$ : Arterial partial pressure ratio of $\mathrm{O}_{2}$ and fraction of inspired $\mathrm{O}_{2}$ mmHg: Millimeters of mercury

rpm: Rotations per minute 
bp: Base pairs

UMIs: Unique molecular identifiers

IPA: Ingenuity Pathway Analysis

FC: Fold-change

FR: Fold-regulation

$\mathrm{N}$ : Sample number

SD: Standard deviation

IV: Intravenous

t.i.d: Three times a day

PO: By mouth

q.d: Once a day

b.i.d: Twice a day

UI: International units

SC: Subcutaneous

PI3K/AKT: Phosphoinositide 3-kinase/protein kinase B

STAT3: Signal transducer and activator of transcription 3

NF- $\kappa \beta$ : Nuclear factor kappa B

ACE2: Angiotensin-converting enzyme 2

ADAM17: A disintegrine and metalloprotease 17

TMPRSS2: Transmembrane serine protease 2

NEAT1: Nuclear paraspeckle assembly ranscript 1

LncRNA: Long non-coding RNA

IFN: Type I interferon

IL-6: Interleukin-6

ARDS: Acute respiratory distress syndrome 


\section{References}

1. Lu H, Stratton CW, Tang YW. Outbreak of pneumonia of unknown etiology in Wuhan, China: The mystery and the miracle. J Med Virol. 2020;92(4):401-2.

2. Guan WJ, Ni ZY, Hu Y, Liang WH, Ou CQ, He JX, et al. Clinical Characteristics of Coronavirus Disease 2019 in China. N Engl J Med. 2020;382(18):1708-20.

3. Coperchini F, Chiovato L, Croce L, Magri F, Rotondi M. The cytokine storm in COVID-19: An overview of the involvement of the chemokine/chemokine-receptor system. Cytokine Growth Factor Rev. 2020;53:25-32.

4. Jean SS, Lee PI, Hsueh PR. Treatment options for COVID-19: The reality and challenges. J Microbiol Immunol Infect. 2020;53(3):436-43.

5. Covid-19 vaccines authorized for emergency use USA 2021 [Available from: https://www.fda.gov/emergency-preparedness-and-response/coronavirus-disease-2019covid-19/covid-19-vaccines; Access in: 05/25/2021

6. $\quad$ Chung JY, Thone MN, Kwon YJ. COVID-19 vaccines: The status and perspectives in delivery points of view. Adv Drug Deliv Rev. 2021;170:1-25.

7. Ji T, Liu Z, Wang G, Guo X, Akbar Khan S, Lai C, et al. Detection of COVID19: A review of the current literature and future perspectives. Biosens Bioelectron. 2020;166:112455.

8. Adeoye J, Thomson P. 'The Double-Edged Sword' - An hypothesis for Covid19-induced salivary biomarkers. Med Hypotheses. 2020;143:110124.

9. Castro R, Luz PM, Wakimoto MD, Veloso VG, Grinsztejn B, Perazzo H. COVID-19: a meta-analysis of diagnostic test accuracy of commercial assays registered in Brazil. Braz J Infect Dis. 2020;24(2):180-7.

10. Jin YH, Zhan QY, Peng ZY, Ren XQ, Yin XT, Cai L, et al. Chemoprophylaxis, diagnosis, treatments, and discharge management of COVID-19: An evidence-based clinical practice guideline (updated version). Mil Med Res. 2020;7(1):41.

11. Pimentel GD, Dela Vega MCM, Laviano A. High neutrophil to lymphocyte ratio as a prognostic marker in COVID-19 patients. Clin Nutr ESPEN. 2020;40:101-2.

12. Mishra PJ, Bertino JR. MicroRNA polymorphisms: the future of pharmacogenomics, molecular epidemiology and individualized medicine. Pharmacogenomics. 2009;10(3):399-416.

13. Correia CN, Nalpas NC, McLoughlin KE, Browne JA, Gordon SV, MacHugh DE, et al. Circulating microRNAs as Potential Biomarkers of Infectious Disease. Front Immunol. 2017;8:118.

14. Marchi R, Sugita B, Centa A, Fonseca AS, Bortoletto S, Fiorentin K, et al. The role of microRNAs in modulating SARS-CoV-2 infection in human cells: a systematic review. Infect Genet Evol. 2021;91:104832.

15. Visacri M, Nicoletti A, Pincinato E, Moriel P. MicroRNAs as biomarkers of coronavirus disease 2019 (COVID19): a scoping review. Research Square; 2021.

16. Falavigna M, Colpani V, Stein C, Azevedo LCP, Bagattini AM, Brito GV, et al. Guidelines for the pharmacological treatment of COVID-19. The task-force/consensus guideline of the Brazilian Association of Intensive Care Medicine, the Brazilian Society of Infectious Diseases and the Brazilian Society of Pulmonology and Tisiology. Rev Bras Ter Intensiva. 2020;32(2):166-96.

17. Agarwal V, Bell GW, Nam JW, Bartel DP. Predicting effective microRNA target sites in mammalian mRNAs. Elife. 2015;4.

18. Kim WR, Park EG, Kang KW, Lee SM, Kim B, Kim HS. Expression Analyses of MicroRNAs in Hamster Lung Tissues Infected by SARS-CoV-2. Mol Cells. 2020;43(11):953-63. 
19. Farr R. Altered microRNA expression in COVID-9 patients enables identification of SARS-CoV-2 infection. In: Rootes C, editor.: Research Square; 2021. 20. Li C, Hu X, Li L, Li JH. Differential microRNA expression in the peripheral blood from human patients with COVID-19. J Clin Lab Anal. 2020;34(10):e23590. 21. Chow JT, Salmena L. Prediction and Analysis of SARS-CoV-2-Targeting MicroRNA in Human Lung Epithelium. Genes (Basel). 2020;11(9).

22. Meerson A, Najjar A, Saad E, Sbeit W, Barhoum M, Assy N. Sex Differences in Plasma MicroRNA Biomarkers of Early and Complicated Diabetes Mellitus in Israeli Arab and Jewish Patients. Noncoding RNA. 2019;5(2).

23. Zou J, Li J, Ji C, Li Q, Guo X. Expression profile of plasma microRNAs in nonsyndromic cleft lip and their clinical significance as biomarkers. Biomed Pharmacother. 2016;82:459-66.

24. Luo J, Wang Y, Lan D, Niu J, Miao J, Dong X, et al. Differential expression of serum microRNAs in glucocorticoid-resistant patients with ulcerative colitis. Int J Clin Exp Pathol. 2018;11(2):936-46.

25. Sabbatinelli J, Giuliani A, Matacchione G, Latini S, Laprovitera N, Pomponio G, et al. Decreased serum levels of the inflammaging marker miR-146a are associated with clinical response to tocilizumab in COVID-19 patients. medRxiv.

2020:2020.07.11.20151365.

26. Garg A, Seeliger B, Derda AA, Xiao K, Gietz A, Scherf K, et al. Circulating cardiovascular microRNAs in critically ill COVID-19 patients. Eur J Heart Fail. 2021.

27. Ackermann M, Verleden SE, Kuehnel M, Haverich A, Welte T, Laenger F, et al. Pulmonary Vascular Endothelialitis, Thrombosis, and Angiogenesis in Covid-19. N Engl J Med. 2020;383(2):120-8.

28. Tang H, Gao Y, Li Z, Miao Y, Huang Z, Liu X, et al. The noncoding and coding transcriptional landscape of the peripheral immune response in patients with COVID19. Clin Transl Med. 2020;10(6):e200.

29. Zhao J, Chen F, Ma W, Zhang P. Suppression of long noncoding RNA NEAT1 attenuates hypoxia-induced cardiomyocytes injury by targeting miR-378a-3p. Gene. 2020;731:144324.

30. Chen YM, Zheng Y, Yu Y, Wang Y, Huang Q, Qian F, et al. Blood molecular markers associated with COVID-19 immunopathology and multi-organ damage. EMBO J. 2020;39(24):e105896.

31. Barbu MG, Condrat CE, Thompson DC, Bugnar OL, Cretoiu D, Toader OD, et al. MicroRNA Involvement in Signaling Pathways During Viral Infection. Front Cell Dev Biol. 2020;8:143.

32. Jafarzadeh A, Nemati M, Jafarzadeh S. Contribution of STAT3 to the pathogenesis of COVID-19. Microb Pathog. 2021;154:104836.

33. Shwetha S, Gouthamchandra K, Chandra M, Ravishankar B, Khaja MN, Das S. Circulating miRNA profile in HCV infected serum: novel insight into pathogenesis. Sci Rep. 2013;3:1555.

34. Hadjadj J, Yatim N, Barnabei L, Corneau A, Boussier J, Smith N, et al. Impaired type I interferon activity and inflammatory responses in severe COVID-19 patients.

Science. 2020;369(6504):718-24.

35. Meffre E, Iwasaki A. Interferon deficiency can lead to severe COVID. Nature. 2020;587(7834):374-6.

36. Hojyo S, Uchida M, Tanaka K, Hasebe R, Tanaka Y, Murakami M, et al. How COVID-19 induces cytokine storm with high mortality. Inflamm Regen. 2020;40:37. 
37. Guo Y, Huang N, Tian M, Fan M, Liu Q, Liu Z, et al. Integrated Analysis of microRNA-mRNA Expression in Mouse Lungs Infected With H7N9 Influenza Virus: A Direct Comparison of Host-Adapting PB2 Mutants. Front Microbiol. 2020;11:1762. 38. Zheng HY, Xu M, Yang CX, Tian RR, Zhang M, Li JJ, et al. Longitudinal transcriptome analyses show robust $\mathrm{T}$ cell immunity during recovery from COVID-19. Signal Transduct Target Ther. 2020;5(1):294.

39. Abu-Izneid T, AlHajri N, Mohammed Ibrahim A, Noushad Javed M, Mustafa Salem K, Hyder Pottoo F, et al. Micro-RNAs in the regulation of immune response against SARS COV-2 and other viral infections. J Adv Res. 2020.

40. Sung SY, Liao CH, Wu HP, Hsiao WC, Wu IH, Jinpu, et al. Loss of let-7 microRNA upregulates IL-6 in bone marrow-derived mesenchymal stem cells triggering a reactive stromal response to prostate cancer. PLoS One. 2013;8(8):e71637. 


\section{Supplementary Materials}

Appendix S1. Quality check of the raw data.

\begin{tabular}{lll}
\hline Sample & \# Reads & \% Bases $\geq \mathbf{Q 3 0}$ \\
\hline MM1 & $11,538,930$ & 93.43 \\
\hline MM2 & $12,451,337$ & 93.38 \\
\hline MM3 & $12,364,410$ & 93.54 \\
\hline SM4 & $14,253,366$ & 93.46 \\
\hline SC2 & $15,052,157$ & 93.59 \\
\hline SC3 & $14,450,638$ & 93.35 \\
\hline SC4 & $13,895,696$ & 93.46 \\
\hline HV1 & $14,155,971$ & 93.51 \\
\hline HV3 & $13,115,540$ & 93.55 \\
\hline HV4 & $15,535,361$ & 93.30 \\
\hline Ab & $13,211,819$ & 93.46 \\
\hline
\end{tabular}

Abbreviations: MM, patient with mild/moderate COVID-19; SC, patient with severe/critical COVID19 ; HV, healthy volunteer. 
Appendix S2. Differentially expressed miRNAs in patients with mild/moderate COVID-19 or severe/critical COVID-19 compared to controls (FR $\geq 1.6$ or FR $\leq$ 1.6).

\begin{tabular}{|c|c|c|c|c|}
\hline \multirow{2}{*}{\multicolumn{5}{|c|}{$\begin{array}{r}\text { miRNA } \\
\text { Mild/moderate COVID-19 } x \text { controls }\end{array}$}} \\
\hline & & & & \\
\hline \multirow[t]{24}{*}{ Downregulated } & miR-4433b-5p & -2.17 & 0.46 & $<0.001$ \\
\hline & miR-5590-5p & -1.91 & 0.52 & 0.002 \\
\hline & miR-224-5p & -1.85 & 0.54 & $<0.001$ \\
\hline & miR-6715b-5p & -1.80 & 0.56 & $<0.001$ \\
\hline & miR-6816-3p & -1.77 & 0.56 & 0.011 \\
\hline & miR-6892-5p & -1.77 & 0.56 & 0.008 \\
\hline & miR-4445-3p & -1.74 & 0.58 & 0.002 \\
\hline & miR-8067 & -1.73 & 0.58 & 0.022 \\
\hline & miR-4726-5p & -1.72 & 0.58 & 0.007 \\
\hline & miR-6841-5p & -1.71 & 0.58 & 0.012 \\
\hline & miR-8054 & -1.71 & 0.58 & 0.022 \\
\hline & miR-4467 & -1.68 & 0.60 & 0.016 \\
\hline & miR-4422 & -1.66 & 0.60 & 0.027 \\
\hline & miR-3607-3p & -1.65 & 0.61 & 0.033 \\
\hline & miR-6073 & -1.65 & 0.61 & 0.002 \\
\hline & miR-3620-5p & -1.64 & 0.61 & 0.021 \\
\hline & miR-7641 & -1.64 & 0.61 & 0.029 \\
\hline & miR-550b-2-5p & -1.63 & 0.61 & 0.023 \\
\hline & miR-421 & -1.62 & 0.62 & 0.010 \\
\hline & miR-548al & -1.62 & 0.62 & 0.025 \\
\hline & miR-181c-3p & -1.61 & 0.62 & 0.036 \\
\hline & miR-3162-3p & -1.61 & 0.62 & 0.016 \\
\hline & miR-767-3p & -1.61 & 0.62 & 0.040 \\
\hline & miR-3689f & -1.60 & 0.62 & 0.042 \\
\hline \multirow[t]{23}{*}{ Upregulated } & miR-4508 & 2.74 & 2.74 & $<0.001$ \\
\hline & miR-187-3p & 2.36 & 2.36 & $<0.001$ \\
\hline & miR-29c-3p & 2.22 & 2.22 & $<0.001$ \\
\hline & $\mathrm{miR}-4532$ & 2.00 & 2.00 & $<0.001$ \\
\hline & miR-3135b & 1.98 & 1.98 & 0.002 \\
\hline & miR-320b & 1.94 & 1.94 & 0.005 \\
\hline & miR-6780b-3p & 1.89 & 1.89 & $<0.001$ \\
\hline & miR-6883-3p & 1.87 & 1.87 & $<0.001$ \\
\hline & miR-4755-3p & 1.82 & 1.82 & $<0.001$ \\
\hline & miR-6511a-3p & 1.79 & 1.79 & 0.015 \\
\hline & miR-4659a-5p & 1.74 & 1.74 & 0.002 \\
\hline & miR-378c & 1.73 & 1.73 & 0.004 \\
\hline & miR-7111-3p & 1.73 & 1.73 & 0.021 \\
\hline & miR-185-5p & 1.72 & 1.72 & 0.020 \\
\hline & miR-4428 & 1.72 & 1.72 & 0.021 \\
\hline & miR-5187-3p & 1.72 & 1.72 & $<0.001$ \\
\hline & miR-521 & 1.72 & 1.72 & 0.024 \\
\hline & miR-320a & 1.71 & 1.71 & 0.025 \\
\hline & miR-4279 & 1.71 & 1.71 & $<0.001$ \\
\hline & miR-378a-3p & 1.69 & 1.69 & 0.026 \\
\hline & miR-1270 & 1.68 & 1.68 & $<0.001$ \\
\hline & let-7e-5p & 1.64 & 1.64 & 0.035 \\
\hline & miR-6785-3p & 1.62 & 1.62 & $<0.001$ \\
\hline
\end{tabular}




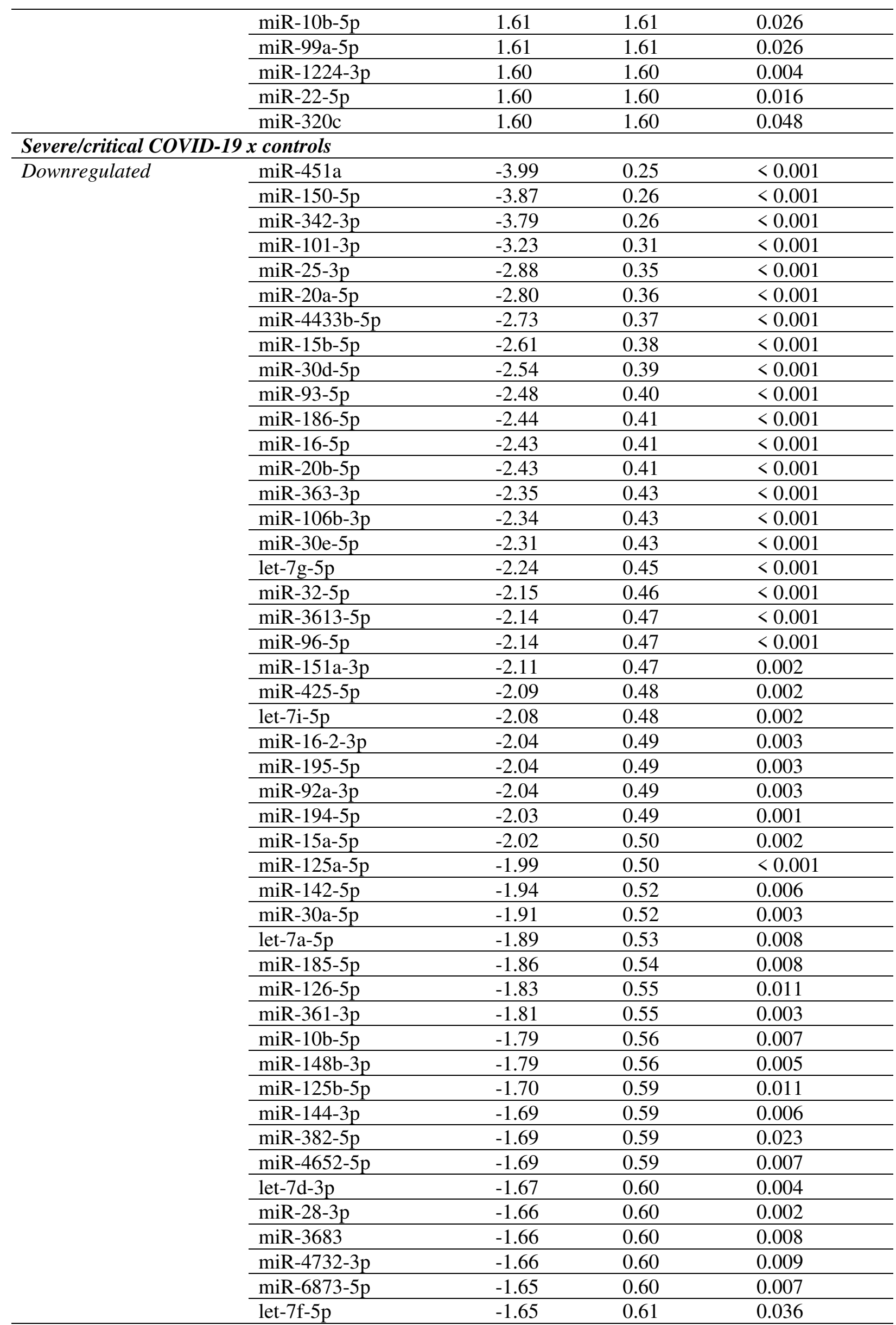




\begin{tabular}{|c|c|c|c|c|}
\hline & miR-19b-3p & -1.65 & 0.61 & 0.004 \\
\hline & miR-22-3p & -1.64 & 0.61 & 0.033 \\
\hline & miR-144-5p & -1.63 & 0.61 & 0.037 \\
\hline & miR-17-5p & -1.63 & 0.61 & 0.004 \\
\hline & miR-579-3p & -1.63 & 0.61 & 0.029 \\
\hline & miR-128-3p & -1.62 & 0.62 & 0.009 \\
\hline & miR-21-5p & -1.62 & 0.62 & 0.029 \\
\hline & let-7d-5p & -1.61 & 0.62 & 0.047 \\
\hline & miR-26a-5p & -1.61 & 0.62 & 0.042 \\
\hline & miR-3182 & -1.61 & 0.62 & 0.040 \\
\hline & miR-485-3p & -1.61 & 0.62 & 0.020 \\
\hline & let-7c-5p & -1.60 & 0.62 & 0.049 \\
\hline & miR-10a-5p & -1.60 & 0.62 & 0.031 \\
\hline \multirow[t]{28}{*}{ Upregulated } & miR-6873-3p & 2.67 & 2.67 & $<0.001$ \\
\hline & miR-4769-5p & 2.32 & 2.32 & $<0.001$ \\
\hline & miR-320c & 2.26 & 2.26 & $<0.001$ \\
\hline & miR-320d & 2.04 & 2.04 & $<0.001$ \\
\hline & miR-7111-3p & 2.03 & 2.03 & 0.003 \\
\hline & miR-320b & 2.01 & 2.01 & 0.003 \\
\hline & miR-6780b-3p & 1.94 & 1.94 & $<0.001$ \\
\hline & miR-6880-3p & 1.91 & 1.91 & $<0.001$ \\
\hline & miR-6511a-3p & 1.78 & 1.78 & 0.016 \\
\hline & miR-6883-3p & 1.78 & 1.78 & $<0.001$ \\
\hline & miR-659-3p & 1.77 & 1.77 & 0.014 \\
\hline & miR-6751-3p & 1.77 & 1.77 & 0.012 \\
\hline & miR-7110-3p & 1.76 & 1.76 & 0.001 \\
\hline & miR-4516 & 1.72 & 1.72 & 0.002 \\
\hline & miR-4784 & 1.71 & 1.71 & $<0.001$ \\
\hline & miR-6753-3p & 1.71 & 1.71 & 0.004 \\
\hline & miR-4326 & 1.70 & 1.70 & $<0.001$ \\
\hline & miR-4755-3p & 1.70 & 1.70 & 0.002 \\
\hline & miR-6791-3p & 1.69 & 1.69 & $<0.001$ \\
\hline & miR-516a-5p & 1.67 & 1.67 & 0.004 \\
\hline & miR-6740-5p & 1.67 & 1.67 & 0.004 \\
\hline & miR-3132 & 1.66 & 1.66 & 0.006 \\
\hline & miR-3915 & 1.66 & 1.66 & 0.002 \\
\hline & miR-4709-3p & 1.65 & 1.65 & 0.006 \\
\hline & miR-483-5p & 1.65 & 1.65 & 0.032 \\
\hline & miR-4701-3p & 1.62 & 1.62 & 0.005 \\
\hline & miR-6126 & 1.62 & 1.62 & 0.018 \\
\hline & miR-760 & 1.61 & 1.61 & 0.038 \\
\hline
\end{tabular}

Abbreviations: FC, fold change; FR, fold regulation; miRNA, miR, microRNA. 
Patients with mild/moderate COVID-19 $x$ controls

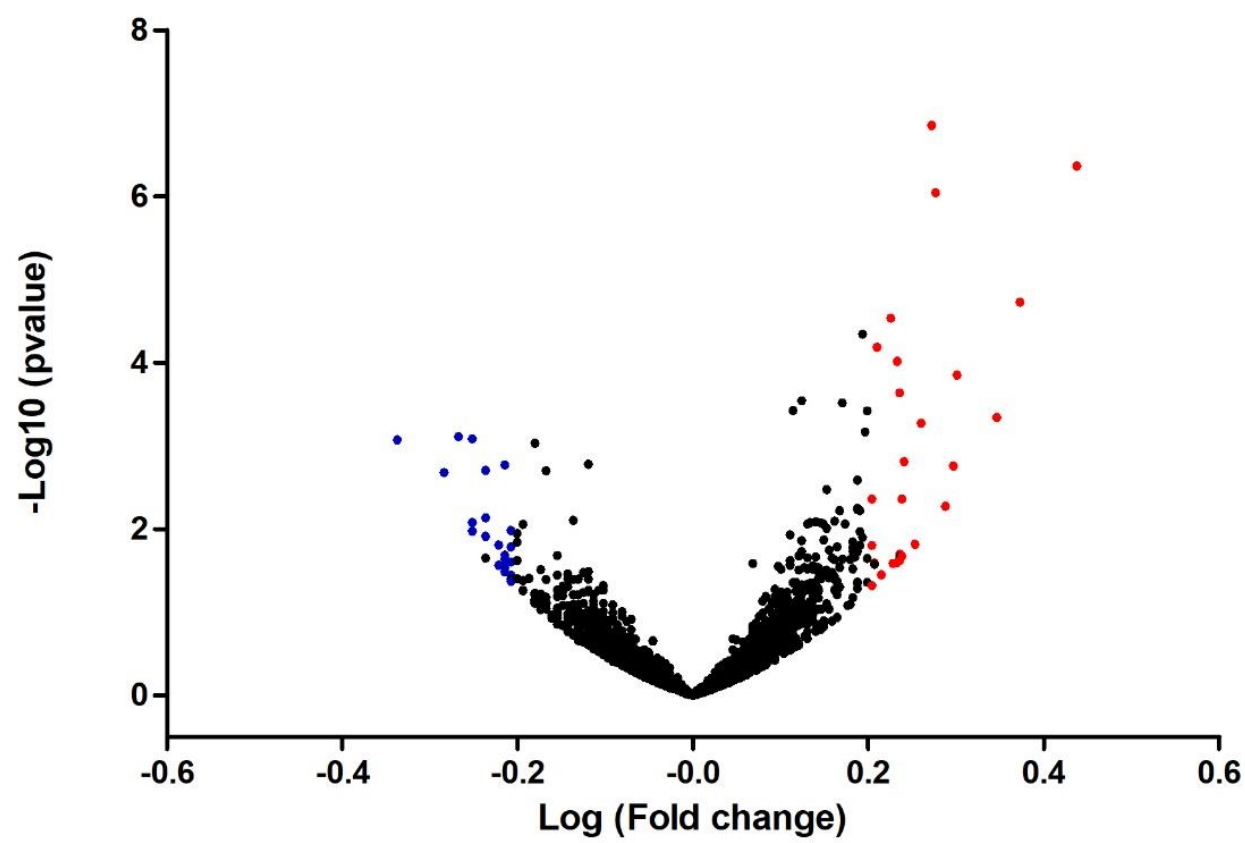

Appendix S3. Differential miRNA expression between patients with mild/moderate COVID-19 and controls (healthy volunteers). The abscissa represents the logarithmic value $\operatorname{logFC}$ of the multiple differences of gene expression between the two groups and the ordinate represents the negative pair value of the p-value of the change in miRNA expression. Each point in the figure represents a miRNA. miRNAs with significant differences are represented by blue and red dots, where the blue dots are downregulated, and the red dots are upregulated. miRNAs without significant differences are represented by black dots. 


\section{Patients with severe/critical COVID-19 $x$ controls}

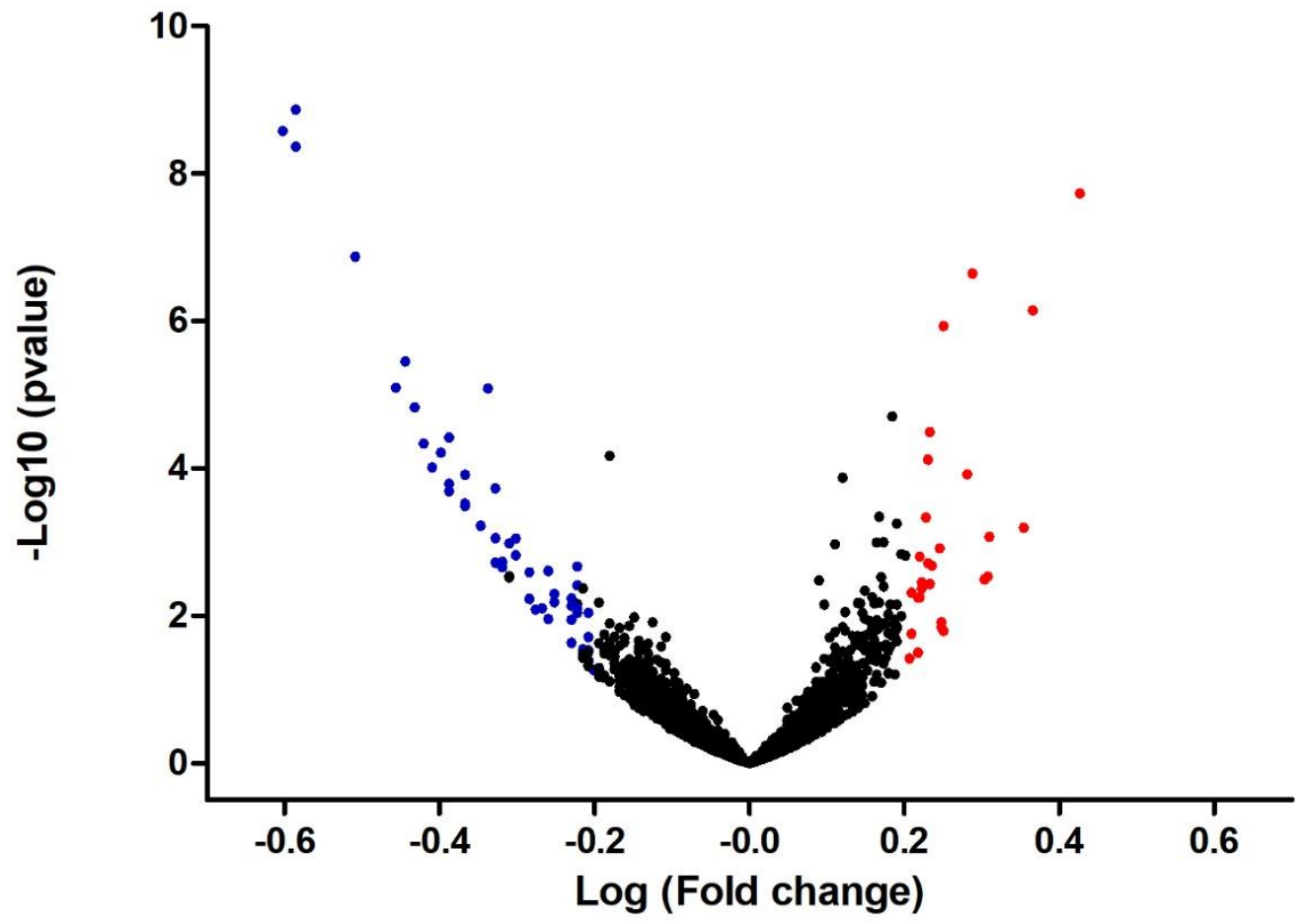

Appendix S4. Differential miRNA expression between patients with severe/critical COVID-19 and controls (healthy volunteers). The abscissa represents the logarithmic value $\log \mathrm{FC}$ of the multiple differences of gene expression between the two groups and the ordinate represents the negative pair value of the p-value of the change in miRNA expression. Each point in the figure represents a miRNA. miRNAs with significant differences are represented by blue and red dots, where the blue dots are downregulated, and the red dots are upregulated. miRNAs without significant differences are represented by black dots. 